\title{
Religiosidad, conductas de riesgo y salud mental en adolescentes de Santiago de Chile*
}

\author{
RAMON FLORENZANO( ${ }^{(1,2,4,6)}$, MACARENA VALDÉS ${ }^{(3)}$, EUGENIO CÁCERES $^{(1,4)}$ SILVIA SANTANDER $^{(5)}$, IVÁN \\ ARMIJO $^{(6)}$, VIVIAN BERGMAN ${ }^{(6)}$ Y ALEJANDRO TRAPP(3)
}

\section{RESUMEN}

El grado de religiosidad y observancia religiosa han sido estudiadas como elementos protectores para diversas conductas de salud. Este estudio analiza la relación entre grado de religiosidad y presencia de uso de sustancias químicas, otras conductas de riesgo auto y hetero-agresivas, en una muestra aleatoria de 252 adolescentes escolarizados en la Región Metropolitana de Santiago de Chile, en base a una aplicación del cuestionario ECRA-2, comparando entre sí los subgrupos auto-calificados como muy religiosos $(n=57)$ con los nada religiosos $(n=50)$. El grupo total tuvo una edad promedio de 17,7 (DS 1,54), de los cuales un 55,9\% eran mujeres. En cuanto a adscripción religiosa, un 72,8\% se identificaban como católicos, un 10,9\% protestantes, un 4,7\% de otra religión y un 11,7\% sin religión. Un 22,6\% se identificó como muy religioso, y un 19,8\% como nada religioso. La comparación presentada contrasta los subgrupos auto-calificados como muy religiosos $(n=57)$ con los nada religiosos $(n=50)$. Un $12,5 \%$ de los adolescentes muy religiosos consumieron marihuana más frecuentemente que una vez al mes, en comparación a un 41,7\% de aquéllos que se consideraban nada religiosos $(8,643 ; 2 g l ; p=0,013)$. Los adolescentes muy religiosos se sentían más frecuentemente tristes y nerviosos que los nada religiosos $\left(22,7 \%\right.$ vs $12,5 \% ; X^{2}=5,175 ; 2 \mathrm{gl}$; $p=0,075)$; informaban menor frecuencia de golpear a otros $(p=0,05)$, menor participación en peleas grupales $(p=0,03)$, menor frecuencia de robo en tiendas $(p=0,08)$ y de copia en pruebas $(p=0,002)$. Los resultados de estudiar la relación entre religiosidad y conductas auto-destructivas (análisis de varianza univariado, $F$ de 3,326 y $R^{2}$ de 0,027 ( $\left.p=0,0001\right)$ señalan que los adolescentes muy religiosos tienen menor tendencia a auto eliminarse. Las conclusiones del análisis concuerdan con la hipótesis del rol protector de la observancia religiosa. Se sugiere profundizar con estudios que exploren en mayor profundidad las variables espiritualidad, religiosidad y observancia como conductas protectoras de la salud mental o general.

Palabras clave: religiosidad, conductas de riesgo, adolescentes.

(1) Instituto de Ciencias de la Familia. Universidad de los Andes. Av. La Plaza 700. Las Condes. Santiago. Chile.rflorenzano@gmail.com

(2) Facultad de Medicina. Universidad de los Andes. Santiago. Chile.

(3) Facultad de Medicina. Universidad de Chile. Santiago. Chile.

(4) Escuela de Psicología. Universidad de los Andes. Santiago. Chile.

(5) Dirección de Asuntos Estudiantiles, Universidad Metropolitana de Ciencias de la Educación. Santiago. Chile.

(6) Facultad de Psicología. Universidad del Desarrollo. Santiago. Chile.

* Trabajo realizado con el apoyo del Fondo de Investigación de la Universidad de los Andes (Proyecto FAIUAndes MED-006-005. 
RELIGIOUSNESS, RISK BEHAVIORS AND MENTAL HEALTH AMONG ADOLESCENTS FROM SANTIAGO, CHILE. (*)

The degree of religiousness and religious observance have been studied as protective elements for various health behaviors. The present study assesses the relation between the degree of religiousness and the use of chemical substances and the presence of aggressive risk behaviors towards oneself or others, in a randomly selected sample of 252 schooled adolescents from the Metropolitan Region of Santiago, Chile. An ECRA-2 questionnaire was used, and the subgroups self-rated as very religious $(n=57)$ and as not religious at all $(n=50)$ were compared. The total group mean age was 17,7 years (SD 1,54), and 55,9\% were female. As for religious ascription, 72,8\% identified themselves as Catholics, 10,9\% as Protestants , 4,7\% as other religion and 11,7\% as without religion. Twenty-two point six percent $(22,6 \%)$ rated themselves as very religious, and 19,8\% as not religious at all. The present comparison contrasts the subgroups self-rated as very religious $(n=57)$ and as not religious at all $(n=50)$. Of the very religious adolescents, $12,5 \%$ consumed marijuana more frequently than once per month, as compared to 41,7\% of those who considered themselves as not religious at all (8,643; $2 \mathrm{gl} ; \mathrm{p}=0$,013). The very religious adolescents felt sad and nervous more commonly than the not religious at all (22,7\% vs 12,5\%; $\left.X^{2}=5,175 ; 2 \mathrm{gl} ; p=0,075\right)$; they reported a lower frequency of beating others $(p=0,05)$, a lesser degree of participation in group fights $(p=0,03)$, a lower frequency of shoplifting $(p=0,08)$ and of cheating behaviors $(p=0,002)$. The results of the assessment of the relationship between religiousness and self-destructive behaviors [univariate analysis of variance, $F$ of 3,326 and $R^{2}$ of 0,027 ( $\left.\left.p=0,0001\right)\right]$ point out that very religious adolescents have less tendency to self destruct. The conclusions of the present analysis are in accordance with the hypothesis of the protective role of religious observance. Further studies exploring deeper the spirituality, religiousness and observance endpoints as protective behaviors for mental or general health are recommended.

Keywords: religiousness, risk behavior, adolescents.

\section{INTRODUCCION}

Después de que por más de un siglo la psicología y el psicoanálisis consideraron los temas espirituales y religiosos como no atingentes al estudio científico de la mente humana ${ }^{1}$, el rol de la religión ha pasado a ser un tema progresivamente importante en la investigación y práctica de la salud mental, durante la última década ${ }^{2}$. Diversos autores han distinguido entre los términos espiritualidad, que se refiere a experiencias trascendentes con un poder superior, del de religiosidad, concepto sociológico que se refiere a un sistema institucionalizado de creencias, valores y actividades para ligarse a ese poder ${ }^{3}$. Finalmente un tercer concepto es el de observancia religiosa, que se refiere a la práctica rutinaria o sistemática de actividades o rituales religiosos. Esta última distinción es especialmente importante en países donde la mayoría de la población dice profesar una fe religiosa, pero donde también se encuentra distancia entre esta profesión verbal y su práctica activa. Este es el caso chileno. Datos de la Encuesta Nacional Bicentenario 2007, realizada por la Pontificia Universidad Católica de Chile Adimark/GFK, basados en una muestra nacional de la población chilena de ambos sexos y más de 18 años y de todos los niveles socio-

\footnotetext{
* Work conducted with the support of the Universidad de Los Andes Research Fund (FAI-UAndes MED-006005 Project)
} 
económicos $(n=2037)^{4}$, muestran que el $86,6 \%$ de los chilenos dijo pertenecer a alguna fe religiosa (católicos $65,5 \%$, otras iglesias cristianas $18,3 \%$, otras Iglesias 2,5\%). Asimismo, un $12,1 \%$ declaró no pertenecer a ninguna religión, y un $1,6 \%$ afirmó ser ateo. Entre los religiosos, declararon asistir a la iglesia o al templo por lo menos una vez a la semana el $21,1 \%$ de los encuestados (14,2\% de los hombres y $26,4 \%$ de las mujeres). En el grupo entre 15 a 24 años de edad, el porcentaje de asistencia más que semanal fue solo de $13,3 \%$, en comparación al $28,1 \%$ del grupo de más de 60 años (Chi2=164,94; 30 df; $p=0,0001)$.

La religiosidad infantil y la de los pre-adolescentes está ligada a las prácticas familiares. Muchos adolescentes a la edad de 12 a 14 asisten en igual porcentaje que sus familias; en los años siguientes hay una disminución en un sub-grupo, y otro que aumenta su observancia religiosa. Diversos autores han estudiados las etapas del desarrollo de la fe y uno de los mejor conocidos, J. Fowler" describió su tercera etapa "sintéticoconvencional" como típica de los jóvenes, con una conformidad inicial a los puntos de vista de otras personas, primero la familia y luego los pares, con un cuestionamiento progresivo de las paradojas y misterios de la religión: los factores cognitivos pasan a ser muy importantes en esta edad. Es también importante la distinción realizada por Gordon Allport ${ }^{6}$ entre la religiosidad extrínseca e intrínseca. Los creyentes intrínsecos creen en profundidad y toman seriamente su fe. Los extrínsecos la ven como un medio para otros fines, generalmente sociales. Muchos jóvenes se tornan en esta etapa creyentes intrínsecos, transformándose su fe en un elemento integral de su auto-imagen y proyecto de vida.

En estudios previos hemos analizado el rol de la familia tanto como factor protector de las conductas de riesgo adolescentes, así como el de la auto-percepción de la funcionalidad familiar. En este trabajo nos centramos en otro elemento contextual que puede influir en éstas, como es la auto-calificación de los adolescentes respecto a su religiosidad, y con indicadores de observan- cia. Para ello hemos analizado la base de datos producida por el estudio Depresión, conductas violentas y adaptación escolar en adolescentes tardios (17-22 años): El rol de la familia, del ambiente académico y de las habilidades sociales, realizado entre los años 2005 y 2006.-

Los objetivos de este estudio buscaron explorar la relación entre auto-percepción de las creencias religiosas, con frecuencias de consumo de tabaco y alcohol y otras conductas de riesgo, así como la presencia de síntomas indicadores de problemas psicopatológicos y búsqueda de ayuda por parte de profesionales de salud mental. Las hipótesis planteadas fueron:

- No hay correlación entre observancia religiosa y consumo frecuente de alcohol o sustancias químicas ilícitas.

- Existe una correlación negativa entre alta observancia religiosa y consumo frecuente de sustancias ilícitas.

- No hay correlación entre creencias religiosas y síntomas de problemas psicopatológicos (ideación suicida, tristeza, ansiedad).

- Existe una correlación negativa entre observancia religiosa y problemas psicopatológicos.

- Existe una correlación negativa entre observancia religiosa y búsqueda de ayuda entre profesionales de la salud mental.

\section{MATERIAL Y MÉTODO}

Como parte del proyecto FAI-UAndes MED006-005 (Depresión, conductas violentas y adaptación escolar en adolescentes tardíos (17-22 años): el rol de la familia, del ambiente escolar y de las habilidades sociales) se encuestaron 263 estudiantes de $3^{\circ}$ Medio de liceos municipalizados de Santiago y Concepción, particulares subvencionados y pagados de Santiago, y de primer año de educación superior técnica y universitaria. Psicólogos especialmente capacitados para ello aplicaron a los estudiantes de cursos completos, los siguientes instrumentos: la Escala de Conductas de Riesgo Adolescentes (ECRA), versión 
validada y adaptada en Chile por nuestro equipo $^{7}$ de un cuestionario, el Minnesota Adolescent Health Survey (MAHS), encuesta preparada por Robert Blum y Cols, en el Programa de Salud del Adolescente de la Universidad de Minnesota ${ }^{8}$ y validada en una muestra de 2160 adolescentes de donde se tradujo al español este instrumento que en su versión original tiene 189 preguntas 9 La modificación realizada en Chile fue producto de un proceso iterativo en el cual se aplicó el cuestionario original a diferentes grupos de adolescentes. La versión utilizada comprende 70 preguntas, que cubren diferentes aspectos de la salud física y emocional de los adolescentes, así como sus antecedentes escolares, nivel socioeconómico, frecuencia de conductas de riesgo tales como consumo de sustancias químicas, prácticas sexuales tempranas, conductas violentas, auto-agresivas y vandálicas, bajo rendimiento y deserción escolares.

a) Los cuestionarios CTF y $\mathrm{CSF}^{10}$, (Como es Tu Familia y Como es Su Familia) en su versión larga. Esta escala fue validada en Chile, Argentina y Colombia y difundida por la Organización Panamericana de la Salud ${ }^{11}$.

Los resultados presentados corresponden a la primera aplicación de la encuesta y los datos se procesaron en SPSS en su versión 15.0.-

\section{RESULTADOS}

La edad promedio de la muestra estudiada fue de 17,7 años (DS 1,54), de los cuales un 55,9\% eran mujeres. Sus padres estaban casados $(66 \%)$, convivía un 4,1\%, un 23,2\% estaba separado, y en un $6 \%$ ambos estaban muertos. Un $70,5 \%$ de los padres trabajaba a tiempo completo o parcial, tal como el $47,9 \%$ de las madres; un $44.4 \%$ estaba en la casa.

En cuanto al consumo de tabaco, no fumaba el $41 \%$; fumaba ocasionalmente un $15,4 \%$, Lo hacía mensual o semanalmente un $12,4 \%$ y diariamente un $31,2 \%$.

Con respecto al consumo de alcohol, no to- maba el $28,2 \%$, ocasionalmente un $35 \%$, mensual un $18,4 \%$ y semanalmente otro $18,4 \%$. No había consumo diario de alcohol.

En cuanto a consumo de marihuana, no fumaba el $85,4 \%$, fumaba ocasionalmente un $3,3 \%$, fumaba mensual, semanal o diariamente un $1,2 \%$. No consumía cocaína el $97,4 \%$ y lo hacía ocasionalmente un $1,7 \%$, consumía semanal o diariamente un $0,8 \%$.

Acerca de conductas hetero-agresivas, había dañado o destruido cosas una a cinco veces un $19,5 \%$, y sobre 6 veces un $10,3 \%$. Había golpeado a otros una a cinco veces un $29,1 \%$, y sobre 6 veces un $4,3 \%$; había estado en peleas grupales una a cinco veces un $19,9 \%$ y sobre 6 veces un $5,6 \%$; había tomado algo de una tienda sin pagarlo una a cinco veces un $10 \%$, y sobre 6 veces un $25,1 \%$; había copiado en pruebas una a cinco veces un $45,9 \%$ y sobre 6 veces un 25,1\%; había hecho la cimarra o capeado clases un $26,4 \%$, y sobre 6 veces un $6,1 \%$. En cuanto a conductas auto agresivas, informaban haber intentado auto-eliminarse en alguna oportunidad 41 sujetos, o sea un $21,2 \%$ de los encuestados.

En cuanto a indicadores de psicopatología, en el último mes había estado extremadamente nervioso un $10 \%$; bastante un $25,1 \%$; un poco el $53,2 \%$ y nada nervioso un $11,7 \%$. En el mismo periodo había estado extremadamente triste, sintiendo que nada valía la pena, un $6,1 \%$; bastante un $16 \%$; un poco un $37,7 \%$ y nada un $40,3 \%$. Asimismo, el 6,9\% declaró haber estado todo el tiempo triste; algunas veces un $84,4 \%$ y nunca un $8,7 \%$. Respecto a todo el tiempo ansioso o preocupado se consideró un $8,2 \%$; algunas veces un $84,4 \%$ y nunca un $7,4 \%$. Por su parte, se había sentido todo el tiempo triste un 34,6\%; algunas veces un $61 \%$; y nunca un $4,3 \%$. Había tenido crisis de nervios en el último año un 18,2\% y hace más de un año un 11,7\%. Había acudido a ver un profesional en el último año un $14,7 \%$ y hace más de un año un 14,3\% adicional.

En cuanto a adscripción religiosa, un 72,8\% se identificaba como católico, un $11,7 \%$ sin religión, un $10,9 \%$ protestantes, y un $4,7 \%$ de otra 
religión. Un 22,6\% se manifestó como muy religioso, y un $19,8 \%$ como nada religioso.

Los adolescentes que se consideraban a si mismos muy religiosos no presentaron una conducta consistente con respecto al consumo de sustancias químicas: consumieron más tabaco, no consumieron cocaína en absoluto, se embriagaron menos frecuentemente y usaron menos marihuana que los que se consideraban nada religiosos, como se ve en la Tabla 1. Estas diferencias alcanzaron significación estadística solo para el consumo de marihuana: un $12,5 \%$ de los adolescentes muy religiosos consumió marihuana más frecuentemente que una vez al mes, en comparación a un $41,7 \%$ de aquellos que se consideraba nada religioso $(8,643 ; 2 \mathrm{gl} ; \mathrm{p}=0,013)$.

Tabla 1. Observancia religiosa y consumo sustancias químicas y conducta sexual en 250 adolescentes encuestados en Regiones Metropolitana y VIII, 2005.

\begin{tabular}{|l|l|l|l|l|}
\hline Conducta de riesgo & $\begin{array}{l}\text { Muy } \\
\text { religioso }\end{array}$ & $\begin{array}{l}\text { Algo } \\
\text { religioso }\end{array}$ & $\begin{array}{l}\text { Nada } \\
\text { religioso }\end{array}$ & $\begin{array}{l}\text { Significación estadística } \\
\text { (Chi cuadrado } \\
\text { de Pearson) }\end{array}$ \\
\hline $\begin{array}{l}\text { Fumar tabaco frecuen- } \\
\text { cia mensual o más }\end{array}$ & $23,2 \%$ & $59,4 \%$ & $17,4 \%$ & $1,543,2$ gl, p=0,4262 \\
\hline $\begin{array}{l}\text { Embriagarse más de } \\
\text { una vez al mes }\end{array}$ & $17,2 \%$ & $54,7 \%$ & $28,1 \%$ & $4,207,2$ gl; $\mathrm{p}=0,121$ \\
\hline $\begin{array}{l}\text { Usa marihuana más } \\
\text { de una vez al mes }\end{array}$ & $12,5 \%$ & $45,8 \%$ & $41,7 \%$ & $\mathbf{8 , 6 4 3 ;}$ 2gl; $\mathbf{p}=\mathbf{0 , 0 1 3}$ \\
\hline $\begin{array}{l}\text { Usa cocaína u otras } \\
\text { drogas más de una vez } \\
\text { al mes }\end{array}$ & $0,0 \%$ & $66,7 \%$ & $33,3 \%$ & 1,$025 ; 2$ gl, $\mathrm{p}=0,599$ \\
\hline
\end{tabular}

Con respecto a la relación entre práctica y salud mental, como se ve en la Tabla 2, los adolescentes muy religiosos se sentían más frecuentemente tristes y nerviosos que los nada religiosos $\left(22,7 \%\right.$ vs $\left.12,5 \% ; X^{2}=5,175 ; 2 \mathrm{gl} ; \mathrm{p}=0,075\right)$; habían sido mas frecuentemente golpeados o maltratados $\left(17,5 \%\right.$ vs $\left.4 \% ; X^{2}=4,732 ; 2 \mathrm{gl} ; \mathrm{p}=0,094\right)$; habían consultado más frecuentemente a especialistas por problemas psicológicos $(40,4 \%$ vs $22 \%$ ) y habían intentado suicidarse menos frecuentemente (14\% vs $20 \%)$. Las diferencias en los dos últimos casos no eran estadísticamente significativas. No hubo tampoco diferencias estadísticas, entre el grado de práctica religiosa y el estado de humor, grado de aburrimiento, de control sobre la propia conducta, de presencia de crisis de pánico.
Al analizar la misma correlación entre autopercepción de mayor frecuencia de prácticas religiosas y conductas hetero-agresivas, los adolescentes muy religiosos informaban, como muestra la Tabla 3, menor frecuencia de golpear a otros $(p=0,05)$, menor participación en peleas grupales $(p=0,03)$, menor frecuencia de robo en tiendas $(p=0,08)$ y de copia en pruebas $(p=0,002)$. No hubo diferencias entre los muy y nada religiosos en conductas vandálicas o en cimarras o capeos fuera de la sala de clases.

Para estudiar en mayor detalle la relación entre autopercepción de práctica religiosa y conductas autodestructivas, al realizar un análisis de varianza univariado, se encuentra un F de 3,326 y $R^{2}$ de 0,027 ( $\left.p=0,0001\right)$. Como se ve en la Tabla 4 , los adolescentes muy religiosos tienen menor 
Tabla 2. Observancia religiosa e indicadores de salud mental en 250 adolescentes encuestados en Regiones Metropolitana y VIII, 2005.

\begin{tabular}{|l|l|l|l|l|}
\hline & $\begin{array}{l}\text { Muy } \\
\text { religioso }\end{array}$ & $\begin{array}{l}\text { Algo } \\
\text { religioso }\end{array}$ & $\begin{array}{l}\text { Nada } \\
\text { religioso }\end{array}$ & $\begin{array}{l}\text { Significación estadística } \\
\text { (Chi cuadrado de Pearson) }\end{array}$ \\
\hline $\begin{array}{l}\text { Triste y nervioso todo } \\
\text { el tiempo }\end{array}$ & $22,7 \%$ & $64,8 \%$ & $12,5 \%$ & $\mathbf{5 , 1 7 5 ; 2}$ gl; p=0,075 \\
\hline $\begin{array}{l}\text { Se ha intentado matar } \\
\text { alguna vez }\end{array}$ & $14 \%$ & $16,8 \%$ & $20 \%$ & $\mathbf{0 , 6 7 8 ; 2 g l ; p = 0 , 7 1 2}$ \\
\hline $\begin{array}{l}\text { Ha consultado espe- } \\
\text { cialista por problemas } \\
\text { psicológicos }\end{array}$ & $40,4 \%$ & $34,0 \%$ & $22,0 \%$ & $\mathbf{4 , 1 9 3 ; 2}$ gl; p=0,123 \\
\hline $\begin{array}{l}\text { Ha sido golpeado o } \\
\text { maltratado }\end{array}$ & $17,5 \%$ & $13,1 \%$ & $4,0 \%$ & $\mathbf{4 , 7 3 2 ; 2}$ gl; $\mathbf{p = 0 , 0 9 4}$ \\
\hline
\end{tabular}

Tabla 3. Correlación de autopercepción de menor religiosidad con conductas hetero-agresivas, 250 adolescentes encuestados en las Regiones Metropolitana y VIII (coeficientes de correlación de Spearman)

\begin{tabular}{|l|l|l|l|l|}
\hline Item & $\begin{array}{l}\text { Coeficiente de } \\
\text { Spearman }\end{array}$ & $\begin{array}{l}\text { Error } \\
\text { típico }\end{array}$ & T Aproximada & $\begin{array}{l}\text { Significación } \\
\text { aproximada }\end{array}$ \\
\hline $\begin{array}{l}\text { Frecuencia con la que ha } \\
\text { destruido cosas }\end{array}$ &, 042 &, 066 &, 673 &, $501(\mathrm{c})$ \\
\hline $\begin{array}{l}\text { Frecuencia con la que ha } \\
\text { golpeado a otros }\end{array}$ &, 123 &, 063 & 1,961 & $\mathbf{, 0 5 1 ( c )}$ \\
\hline $\begin{array}{l}\text { Frecuencia de peleas } \\
\text { entre grupos }\end{array}$ &, 137 &, 061 & 2,189 & $\mathbf{, 0 3 0 ( c )}$ \\
\hline $\begin{array}{l}\text { Frecuencia de robo en } \\
\text { tiendas }\end{array}$ &, 111 &, 062 & 1,777 & $\mathbf{, 0 7 7 ( c )}$ \\
\hline $\begin{array}{l}\text { Frecuencia de copia en } \\
\text { pruebas }\end{array}$ &, 191 &, 064 & 3,076 & $\mathbf{, 0 0 2 ( c )}$ \\
\hline $\begin{array}{l}\text { Frecuencia de cimarras o } \\
\text { capeos }\end{array}$ &,- 034 &, 063 &,- 532 &, $595(\mathrm{c})$ \\
\hline
\end{tabular}

c Basada en la aproximación normal.

tendencia a autoeliminarse, lo que concuerda con la hipótesis del rol protector de la observancia religiosa. Analizamos luego el rol de la separación parental con los indicadores de salud mental antes estudiados. La matriz de correlación no paramétrica que se ve en la Tabla 5 muestra una correlación negativa entre mayor religiosidad del adolescente y separación parental (Coeficiente de correlación de Spearman -0,147; $\mathrm{p}=0,02$ ), así como entre mayor religiosidad y consultar a especialistas de salud mental (Coeficiente de correlación de Spearman -0,231; $\mathrm{p}=0,0001$ ). 
Religiosidad, conductas de riesgo y salud mental en adolescentes... - Ramón Florenzano et al

Tabla 4. Correlación entre autopercepción de menor religiosidad e intentos suicidas (Análisis de varianza: efectos intersujetos), en 41 escolares que los presentan, Regiones Metropolitana y VIII, 2005.

\begin{tabular}{|l|l|l|l|l|l|}
\hline Fuente & $\begin{array}{c}\text { Suma de cuadrados } \\
\text { tipo III }\end{array}$ & Gl & \multicolumn{1}{|c|}{$\begin{array}{c}\text { Media } \\
\text { cuadrática }\end{array}$} & F & Significación \\
\hline Modelo corregido & $2,754(\mathrm{a})$ & 2 & 1,377 & 3,326 &, 038 \\
\hline Intersección & 85,427 & 1 & 85,427 & 206,393 &, 0001 \\
\hline $\begin{array}{l}\text { Ha intentado } \\
\text { matarse }\end{array}$ &, 353 & 1 &, 353 &, 854 &, 356 \\
\hline Error & 99,751 & 241 &, 414 & & \\
\hline Total & 1035,000 & 244 & & & \\
\hline Total corregida & 102,504 & 243 & & & \\
\hline
\end{tabular}

a $\mathrm{R}$ cuadrado $=, 027(\mathrm{R}$ cuadrado corregida $=, 019)$

Tabla 5. Correlación entre considerarse muy religioso, indicadores de salud mental y situación matrimonial de los padres (Matriz de correlaciones no paramétricas de Spearman), 250 adolescentes Regiones Metropolitana y VIII, 2005.

\begin{tabular}{|c|c|c|c|c|c|}
\hline & & $\begin{array}{l}\text { Cómo se } \\
\text { considera } \\
\text { frente a } \\
\text { religión }\end{array}$ & $\begin{array}{l}\text { Se ha sen- } \\
\text { tido triste o } \\
\text { nervioso(a) } \\
\text { todo el tiempo }\end{array}$ & $\begin{array}{l}\text { Ha consultado a un } \\
\text { especialista por pro- } \\
\text { blemas emocionales } \\
\text { o psicológicos alguna } \\
\text { vez }\end{array}$ & $\begin{array}{l}\text { Padres sepa- } \\
\text { rados actual- } \\
\text { mente (no } \\
\text { viven juntos) }\end{array}$ \\
\hline \multirow[t]{2}{*}{$\begin{array}{l}\text { Cómo se considera } \\
\text { frente a religión }\end{array}$} & $\begin{array}{l}\text { Coeficiente } \\
\text { de correla- } \\
\text { ción }\end{array}$ & 1,000 & ,087 & ,125(*) &,$- 147(*)$ \\
\hline & $\begin{array}{l}\text { Sig. (bilate- } \\
\text { ral) }\end{array}$ & . & , 170 & ,047 & ,020 \\
\hline \multirow[t]{2}{*}{$\begin{array}{l}\text { Se ha sentido triste } \\
\text { o nervioso(a) todo } \\
\text { el tiempo }\end{array}$} & $\begin{array}{l}\text { Coeficiente } \\
\text { de correla- } \\
\text { ción }\end{array}$ & ,087 & 1,000 &, $231(* *)$ &,- 053 \\
\hline & $\begin{array}{l}\text { Sig. (bilate- } \\
\text { ral) }\end{array}$ &, 170 & . &, $0001(*)$ & ,409 \\
\hline \multirow{2}{*}{$\begin{array}{l}\text { Ha consultado a } \\
\text { un especialista por } \\
\text { problemas emocio- } \\
\text { nales o psicológi- } \\
\text { cos alguna vez }\end{array}$} & $\begin{array}{l}\text { Coeficiente } \\
\text { de correla- } \\
\text { ción }\end{array}$ &, $125\left(^{*}\right)$ &, $231(* *)$ & 1,000 & ,101 \\
\hline & $\begin{array}{l}\text { Sig. (bilate- } \\
\text { ral) }\end{array}$ & ,047 & ,000 & . & ,112 \\
\hline \multirow[t]{2}{*}{$\begin{array}{l}\text { Padres separados } \\
\text { actualmente (no } \\
\text { viven juntos) }\end{array}$} & $\begin{array}{l}\text { Coeficiente } \\
\text { de correla- } \\
\text { ción }\end{array}$ &,$- 147(*)$ &,- 053 & ,101 & 1,000 \\
\hline & $\begin{array}{l}\text { Sig. (bilate- } \\
\text { ral) }\end{array}$ &, 020 & ,409 & ,112 & . \\
\hline
\end{tabular}

* La correlación es significativa al nivel 0,05 (bilateral).

** La correlación es significativa al nivel 0,01 (bilateral). 
Para analizar en profundidad la correlación con conductas hetero-destructivas, la matriz de correlación no paramétrica que muestra la Tabla 6 encuentra correlaciones significativas entre falta de religiosidad y mayor frecuencia de copia en pruebas $(\mathrm{p}=0,02)$; conductas vandálicas y hacer la cimarra $(\mathrm{p}=0,0001)$; conductas vandáli- cas y golpear a otros $(\mathrm{p}=0,0001)$; conductas vandálicas y robar en tiendas $(\mathrm{p}=0,0001)$; conductas vandálicas y copiar en pruebas $(\mathrm{p}=0,0001)$. El robar en tiendas se correlacionó significativamente con golpear a otros $(p=0,0001)$; con copiar en pruebas $(\mathrm{p}=0,001)$; y con hacer la cimarra $(\mathrm{p}=0,001)$.

Tabla 6. Correlación entre frecuencia de práctica religiosa y conductas hetero-agresivas (matriz de correlaciones no paramétricas de Spearman) en 250 adolescentes de las regiones Metropolitana y VIII, 2005.

\begin{tabular}{|c|c|c|c|c|c|c|c|}
\hline & & $\begin{array}{l}\text { Baja } \\
\text { religiosidad }\end{array}$ & Vandalismo & $\begin{array}{l}\text { Golpea } \\
\text { a otros }\end{array}$ & $\begin{array}{l}\text { Robo en } \\
\text { tiendas }\end{array}$ & $\begin{array}{l}\text { Copia en } \\
\text { prueba }\end{array}$ & $\begin{array}{l}\text { Cimarras } \\
\text { o capeos } \\
\text { de clase }\end{array}$ \\
\hline \multirow[t]{2}{*}{$\begin{array}{l}\text { Baja } \\
\text { religiosidad }\end{array}$} & $\begin{array}{l}\text { Coeficiente } \\
\text { de correla- } \\
\text { ción }\end{array}$ & 1,000 &, 042 & ,123 & ,111 &, $191(* *)$ &,- 034 \\
\hline & Sig. & . & ,501 &, 051 &, 077 & ,002 & ,595 \\
\hline \multirow[t]{2}{*}{ Vandalismo } & $\begin{array}{l}\text { Coeficiente } \\
\text { de correla- } \\
\text { ción }\end{array}$ &, 042 & 1,000 &, $372(* *)$ &, $368(* *)$ &, $387(* *)$ &, $296(* *)$ \\
\hline & Sig & ,501 & . &, 000 &, 000 &, 000 &, 000 \\
\hline \multirow[t]{2}{*}{$\begin{array}{l}\text { Golpea a } \\
\text { otros }\end{array}$} & $\begin{array}{l}\text { Coeficiente } \\
\text { de correla- } \\
\text { ción }\end{array}$ & ,123 &, $372(* *)$ & 1,000 &, $338(* *)$ &, $275(* *)$ &, $315(* *)$ \\
\hline & Sig. &, 051 &, 000 & . &, 000 &, 000 &, 000 \\
\hline \multirow[t]{2}{*}{$\begin{array}{l}\text { Robo en } \\
\text { tiendas }\end{array}$} & $\begin{array}{l}\text { Coeficiente } \\
\text { de correla- } \\
\text { ción }\end{array}$ & ,111 &, $368(* *)$ &, $338(* *)$ & 1,000 &, $385(* *)$ &, $239(* *)$ \\
\hline & Sig. & ,077 & ,000 & ,000 & . & ,000 & ,000 \\
\hline \multirow[t]{2}{*}{$\begin{array}{l}\text { Copia en } \\
\text { pruebas }\end{array}$} & $\begin{array}{l}\text { Coeficiente } \\
\text { de correla- } \\
\text { ción }\end{array}$ &, $191(* *)$ &, $387(* *)$ &, $275(* *)$ &, $385(* *)$ & 1,000 &, $280(* *)$ \\
\hline & Sig. &, 002 &, 000 &, 000 &, 000 & . &, 000 \\
\hline \multirow[t]{2}{*}{$\begin{array}{l}\text { Cimarras o } \\
\text { capeos de } \\
\text { clase }\end{array}$} & $\begin{array}{l}\text { Coeficiente } \\
\text { de correla- } \\
\text { ción }\end{array}$ &,- 034 &, $296(* *)$ &, $315(* *)$ &, $239(* *)$ &, $280(* *)$ & 1,000 \\
\hline & Sig. &, 020 & ,091 & ,337 &, 456 &, 749 & ,983 \\
\hline
\end{tabular}

** La correlación es significativa al nivel 0,01 (bilateral).

* La correlación es significativa al nivel 0,05 (bilateral). 


\section{CONCLUSIONES}

Entre las limitaciones del estudio debemos mencionar que la percepción del funcionamiento familiar, del consumo de sustancias químicas, y de la auto-calificación de observancia religiosa fueron hechas por auto reporte: si bien los cuestionarios aplicados fueron respondidos por los mismos jóvenes en forma anónima, los resultados pueden ser poco confiables por factores influyentes tales como deseabilidad social, fallas mnémicas y fatiga durante la aplicación de los cuestionarios. Una segunda limitación es el tamaño de la muestra y su representatividad, que al centrarse en estudiantes de tercero medio y primero de universidad o instituto técnico de la carrera de enfermería, sesgó la recolección hacia el sexo femenino. Esto hace que los resultados no sean generalizables a todos los estudiantes escolarizados de la Región Metropolitana y tampoco a ámbitos territoriales mayores.

Al comparar las prevalencias con el Séptimo Estudio Nacional de Drogas en Población General de Chile realizado el 2006, que consideró a un total de 16.807 personas entre 12 y 64 años de edad, hombres y mujeres, de todos los niveles socioeconómicos, que habitan en 91 ciudades con población urbana de 30 mil habitantes o más ${ }^{12}$, encontramos cifras de consumo de tabaco, alcohol y marihuana cercanas a las informadas para ese grupo de edad por CONACE. Asimismo, confirmamos la asociación encontrada por ellos entre consumo de sustancias químicas y otras conductas de riesgo, tales como vandalismo, involucrarse en riñas, robo y otras conductas antisociales. También corroboramos los planteamientos del Séptimo Estudio Nacional en relación a la relación con funcionamiento familiar. Esta asociación ha sido también informada recientemente por un estudio de la Universidad Católica de Chile ${ }^{13}$, de modo que ese estudio confirma estudios previos, tanto nuestros como otros más recientes ligando la funcionalidad familiar con la frecuencia de conductas de riesgo.

En relación a la religiosidad, confirmamos que se trata de un constructo complejo y mul- tidimensional, como lo señalan Kendler et $\mathrm{al}^{14}$. El cuestionario utilizado sólo explora la auto percepción de observancia religiosa y la práctica religiosa reportada por el sujeto. Por una parte confirmamos que los sujetos más religiosos consumen menos marihuana y alcohol (no así tabaco), lo que ha sido informado en diversos estudios tanto internacionales, latinoamericanos como chilenos ${ }^{15}$. Por otra parte, los adolescentes más religiosos reportan más tristeza y síntomas relacionados con depresión, así como consultan más a especialistas de salud mental que los menos o nada religiosos.

Los intentos suicidas son menores en los practicantes religiosos que en los no religiosos, pudiendo afirmarse que la religiosidad protege de la auto-agresión suicida. Las conductas hetero-agresivas en su mayoría también presentan diferencias significativas asociándose menor práctica con más beligerancia con otros, robos y problemas conductuales en la escuela.

En futuros estudios, fuera de aumentar el número de casos y la representatividad de la muestra, es necesario profundizar entre distintos aspectos de la religiosidad, separando el rol de la familia y de ésta. En el plano teórico, es importante distinguir el efecto de la creencia en Dios y sus características (el aspecto ontológico, que difiere en cada credo religioso), de la observancia religiosa (el aspecto sociológico, con sus elementos de sistema de apoyo y de control social). Los últimos parecen ser especialmente relevantes en el control de conductas de riesgo, sean de uso de sustancias químicas como de síntomas emocionales. Las diferencias encontradas con respecto a uso de alcohol, tabaco y drogas ilegales pueden servir para distinguir entre las variables anteriores. Por ejemplo, el estudio de Chen antes citado encontró que si bien la observancia de cualquier credo disminuyó, en su estudio en siete países latinoamericanos, el consumo de cualquier sustancia química, hubo una variabilidad con respecto al uso de alcohol por católicos y protestantes.

Trabajo invitado 


\section{REFERENCIAS}

1 FLORENZANO $\mathrm{R} \quad \mathrm{Y}$ VALDÉS $\mathrm{M}$. $\mathrm{El}$ Adolescente y sus Conductas de Riesgo. Tercera Edición. Ediciones Universidad Católica de Chile, Santiago, 2005.

2 ORLINSKY D. Religious and spiritual experiences of psychotherapists. Book of Abstracts, 39 $9^{\text {th }}$ International Meeting Society for Psychotherapy Research (SPR). Barcelona, España, Junio 18-21, 2008.

3 KELLY EW. Spirituality and religiosity in counseling and psychotherapy. Alexandria, Virginia, American Counseling Association, 2002.

4 INSTITUTO DE SOCIOLOGIA PONTIFICIA UNIVERSIDAD CATÓLICA DE CHILE Y CEP ADIMARK. Encuesta Nacional Bicentenario 2007.

5 FOWLER FJ, NIPKOW KE Y SCHWEITZER $F$ (EDS). Stages of Faith and Religious Development. New York, Crossroads, 1991.

6 ALLPORT GW. The Individual and his Religion. New York, Macmillan 1950.

7 MARCHANDÓN, A. FLORENZANO, R., PINO, P. Adaptación en Chile de un cuestionario para medir conductas de riesgo en adolescentes. Actas del II Encuentro de Investigadores Médico-Sociales sobre la Juventud, Santiago de Chile, 1992.

8 BLUM, R. Technical report on the Adolescent Health Survey. Adolescent Health Database Project, Universidad de Minnesota, Minnesota, 1989.
9 HERNÁNDEZ, A, BEUHRING, T, BERNER, E., BLUM, R., FLORENZANO, R., LAMMERS, SERRANO, C. \& VALDÉS, M. 1996. Familia y Adolescencia: Indicadores de Salud. Manual de aplicación de Instrumentos. Organización Panamericana de la Salud, Washington D.C.

10 VALDÉS M, RODRÍGUEZ J, SERRANO T. Adaptación de la Escala Abreviada del Instrumento "Como es tu Familia-Breve" y "Como es Su Familia-Breve". En: REf anterior.

11 SANTIS R, HIDALGO CG, HAYDEN V, ANSELMO E, RODRÍGUEZ J, CARTAJENAF, DREYSE J, TORRES R. Consumo de sustancias y conductas de riesgo en consumidores de pasta base de cacaína no consultantes a servicios de rehabilitación Rev Méd Chile 2007; 135: 45-53.

12 CONACE. Séptimo Estudio Nacional de Drogas en Población General de Chile. Ministerio del Interior, Chile, 2006.

13 SANTANDER S, ZUBAREW T, SANTELICES L, ARGOLLO A, CERDA J, BORQUEZ M. Influencia de la familia como factor protector de conductas de riesgo en escolares chilenos. Rev Med Chile, Marzo 2008.

14 KENDLER KS, LIO XQ, GARCNER CO, MCCULLOUGH ME, LARSON D Y PRESCOTT CA. Dimensions of Religiosity and Their Relationship to Lifetime Psychiatric and Substance Abuse Disorders. Am J Psychiatry 2003: 160:490-503.

15 CHEN CY, DORMITZER CM, BEJARANO J Y ANTHONY JC. Religiosity and Earliest Stages of Adolescent Drug Involvement in Seven Countries in Latin America. Amer C. Epidemiol. 2004, 159:1160-1188.

Usted puede comentar éste y otros artículos publicados en la Revista Chilena de Salud Pública, enviando un correo electrónico a revistasp@med.uchile.cl 